Ignition X-Ray Imager for Laser-Fusion Research at the National Ignition Facility

R. Tommasini, T. W. Phillips, J. A. Koch

October 13, 2005

4th International Fusion Sciences \& Applications Biarritz, France September 4, 2005 through September 9, 2005 
This document was prepared as an account of work sponsored by an agency of the United States Government. Neither the United States Government nor the University of California nor any of their employees, makes any warranty, express or implied, or assumes any legal liability or responsibility for the accuracy, completeness, or usefulness of any information, apparatus, product, or process disclosed, or represents that its use would not infringe privately owned rights. Reference herein to any specific commercial product, process, or service by trade name, trademark, manufacturer, or otherwise, does not necessarily constitute or imply its endorsement, recommendation, or favoring by the United States Government or the University of California. The views and opinions of authors expressed herein do not necessarily state or reflect those of the United States Government or the University of California, and shall not be used for advertising or product endorsement purposes. 
The headers will be insert by the Publisher

The headers will be insert by the Publisher

The headers will be insert by the Publisher

\title{
Ignition X-Ray Imager for Laser-Fusion Research at the National Ignition Facility
}

\author{
Riccardo Tommasini, Thomas W. Phillips, and Jeffrey A. Koch \\ Lawrence Livermore National Laboratory, \\ P.O. Box 5508, Livermore, California 94550 USA
}

\begin{abstract}
X-ray imaging will be an important diagnostic tool for inertial confinement fusion (ICF) research at the National Ignition Facility (NIF). However, high neutron yields will make x-ray imaging much more difficult than it is at current smaller facilities. We analyze the feasibility and performance of an Ignition X-Ray Imager to be used on cryogenic DT implosions at NIF. The system is intended to provide time-integrated, broadband, moderate-energy x-ray core images of imploding ICF capsules. Highly magnified, spectrally-filtered images created using an array of pinholes placed close to the target will be projected onto a scintillator placed at the target chamber wall. A telescope will be used to relay the scintillator emission to a distant optical detector that is time-gated in order to minimize backgrounds, in particular from neutrons. The system is optimized with respect to spatial-resolution, signal-to-background and signal-to-noise ratios.
\end{abstract}

\section{Introduction}

We present a feasibility study and design of a High-Energy X-Ray Imager (HEXRI) to be used on cryogenic DT implosions at the National Ignition Facility. Even though the system is essentially based on a simple X-ray pinhole camera, the extremely high anticipated neutron yields and X-rays generated by laser plasma instabilities (LPI) will make X-ray imaging much more difficult than it is at current smaller facilities. The HEXRI's primary mission will be to record X-ray core emission images of preignition cryogenic DT implosions to aid in recovery from possible failures to achieve ignition. Therefore the critical parameters to be measured are the core size and shape, using broadband imaging around $9-10 \mathrm{keV}$. A secondary mission will be to provide hot-spot electron temperature and density, by cross-comparison of multiple higher-energy (10-20 keV) differentially filtered x-ray core images. Another secondary mission will be to observe main-fuel areal-density spatial variations by crosscomparison of multiple lower-energy $(<10 \mathrm{keV})$ differentially filtered $\mathrm{x}$-ray core images that use core emission to backlight cold main-fuel plasma. The primary requirements for such a system are: a spatial resolution better than $10 \mu \mathrm{m}$ for energies between 9 and $20 \mathrm{keV}$; temperature and density profiles accurate to $20 \%$ near the center of the core ; adequate (better than 5) signal-to-noise (SNR) and signal-to-background (SBR) ratios; a field of view (FOV) larger than $100 \mu \mathrm{m}$; operability over a neutron yield range between $1 \mathrm{e} 15$ and $1 \mathrm{e} 17$. 


\section{System description}

HEXRI $^{(1)}$ will be a time-integrated pinhole/scintillator system, converting x-rays to visible light and relaying the images to a distant detector. Figure 1 shows the basic setup of the system with the definition of the various parameters. The images will be created using an array of pinholes mounted, close to target, on the ignition cryogenic target positioner. The highly magnified $(\sim 100 x)$ images of implosion cores will be projected onto a scintillator placed at the target chamber wall, and filters placed in front of the scintillator will restrict the detected x-ray spectrum. A telescope system will then be used to relay the scintillator emission to a distant detector that is timegated in order to minimize neutron-induced backgrounds. The emitted neutron distribution, peaking at $14 \mathrm{MeV}$, requires detector time-gating to eliminate the latearriving background, on times shorter than $50 \mathrm{~ns}$. This fixes an upper value for the

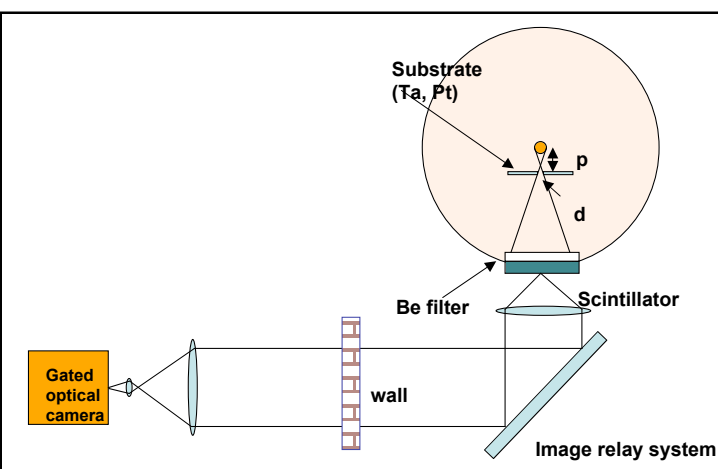

Figure 1: Sketch of the simplified set up for the HEXRI. scintillator decay time. On the other hand the phosphor is required to have radiation hardness high enough to sustain tens of NIF shots without noticeable changes in detection properties. These two constraints appear to narrow our choices to $\mathrm{BaF}$, LSO and GSO as scintillating materials. The spatial resolution $\sigma$ on the object is assumed to be the convolution of the geometrical resolution and the diffraction point spread function ${ }^{(2)}: \sigma^{2}=[d(1+1 / M)]^{2}$ $+(2.44 \lambda \mathrm{p} / \mathrm{d})^{2}$, where $\mathrm{M}$ is the magnification. The spatial resolution has been optimized assuming

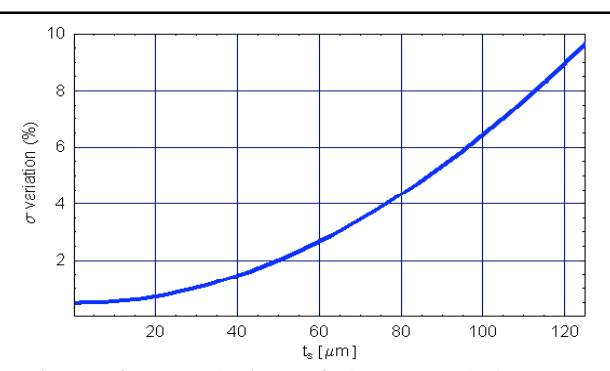

Figure 2: Resolution of the cascaded system: variation, with respect to the pure pinhole resolution. F-number $=2$. At $\sim 100 \mu \mathrm{m}$ the resolution is still very good $(6.2 \mathrm{~mm}$ vs. 5.8 $\mathrm{mm})$. the minimum possible pinhole-target distance, $50 \mathrm{~mm}$, and the scintillator at the chamber wall. Under these conditions a resolution of $5.8 \mu \mathrm{m}$ at 9 $\mathrm{keV}$ is obtained for a pinhole diameter of $4 \mu \mathrm{m}$ and a magnification of $\sim 100 x$. The FOV will be limited only by the detector size. The primary sources of image blur in the whole cascaded system are radiation scattering in the scintillator and depth-offocus of the optical system. Both increase with the scintillator thickness, $\mathrm{t}_{\mathrm{s}}$. Fig. 2 shows that, as far as resolution is of concern, we can accept scintillator thicknesses up to $\sim 120 \mu \mathrm{m}$. The expected $\mathrm{x}$-ray source brightness has been estimated from target design simulations ${ }^{(3)}$ to be $10^{16} \mathrm{~W} / \mathrm{cm}^{2} \mathrm{Sr} \mathrm{keV}$ at 9

$\mathrm{keV}$, with duration of $100 \mathrm{ps}$.

The scintillator output has been calculated considering a $10 \mu \mathrm{m}$ thick Au hohlraum and a $7 \mathrm{~mm}$-thick Be filter. X-rays passing through the pinhole substrate generate backgrounds, so that the thickness and composition of the substrate needs to be considered.

The system will produce a 10x10 array of differentially filtered images in order to obtain hot-spot temperature and density maps. This, together with the magnification, will require a scintillator with a transverse dimension of about $15 \mathrm{~cm}$. The scintillator area and the size of the gated optical camera will then determine the final magnification of the relay optical system. Figure 3 reports the spectrumintegrated signal-to-noise ratio (SNR, from statistics) and the signal-to- background ratio (SBR, from $\mathrm{X}$-rays passing the pinhole substrate), together with the loss (\%) in resolution at the source plane vs. scintillator thickness, of the full-cascaded system, i.e. including the image relay optics (F\#2) and 


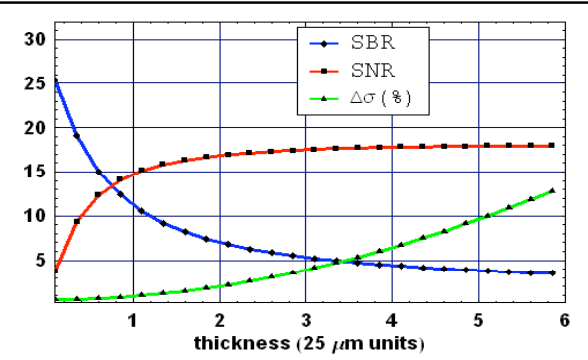

Figure 4

SNR, SBR and loss in resolution vs. thickness for a GSO scintillator.

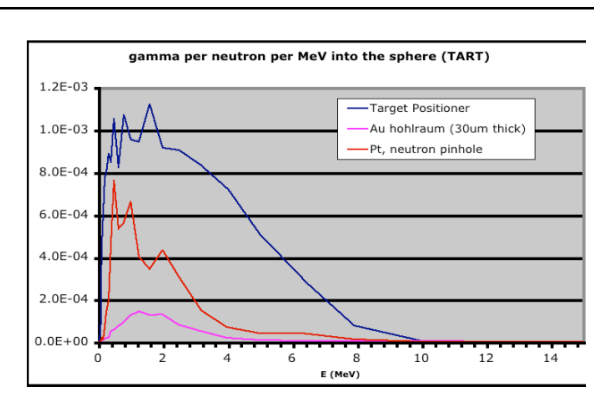

\section{Figure 5}

Monte Carlo simulations of gamma rays spectrum for the main sources inside the NIF target chamber.
CCD. The pinhole substrate is $\mathrm{Pt}, 75 \mu \mathrm{m}$ thick, the scintillator is GSO, $25 \mu \mathrm{m}$ thick. The detection process in the scintillator has been assumed to follow Poissonian statistics ${ }^{(4)}$. The SNR can be improved by using a thinner Be filter, $1 \mathrm{~mm}$ vs. $7 \mathrm{~mm}$ thickness gives a factor of $\sim 1.5$ increase, and a reflection coating on the front side of the scintillator, giving an increase of a factor of $\sqrt{ } 2$. An important source of background are gamma rays from neutron-gamma reactions. The results from Monte Carlo simulations $\left(\mathrm{TART}^{(5)}\right.$ ) of various gamma sources inside the NIF target chamber are shown in Fig.5, reporting the spectrum of the gamma rays incident onto the scintillator. As a result the major sources of gamma background are the target positioner and the pinhole for neutron imaging.

The transport of gamma rays into the scintillator has been calculated using a dedicated Monte Carlo code. The resulting signal-to-background levels for the three scintillators under consideration is found to be $\sim 6$, assuming a neutron yield of $1 \mathrm{E} 17$ and a scintillator thickness of $25 \mu \mathrm{m}$. The background from LPI X-rays is currently under evaluation. Preliminary calculations using a thick target bremsstrahlung formula ${ }^{\left({ }^{6}\right)}$ show that assuming a conversion efficiency of the laser energy into hot electrons of $1 \%$ results in a SBR of about 2. Doubling the pinhole substrate thickness increases the SBR up to 5.

\section{Conclusions}

Using a pinhole about $4 \mu \mathrm{m}$ in diameter a resolution of $5.8 \mu \mathrm{m}$ is achieved at $9 \mathrm{keV}$, limited by restrictions in the pinhole positioning. The resolution varies between 8.5 and $4.5 \mu \mathrm{m}$ in the $5-20 \mathrm{keV}$ spectral range. Signal/Background (SBR) and Signal/Noise (SNR) ratios have been estimated to be adequate for available scintillators showing fast enough decay time and suitable radiation hardness, namely $\mathrm{BaF}$, GSO and LSO. We are currently developing a more adequate Monte Carlo simulation to account for background from n-gamma reactions and LPI X-rays.

\section{Acknowledgments}

This work was performed under the auspices of the U.S. Department of Energy by University of California, Lawrence Livermore National Laboratory under Contract W-7405-Eng-48.

\section{References}

[1] R. Tommasini and J.A. Koch, LLNL Report, UCRL-TR-211667, (2004).

[2] J. A. Koch et al., Appl. Opt. 37 (10) 1784 (1998).

[3] Steven Haan, LLNL, private communication.

[4] Swank, J. Appl. Phys. 44, 4199 (1973)

[5] E.F. Plechaty and R.E. Dye, UCID-17026, , Lawrence Livermore National Laboratory (1992)

[6] W. Kruer, "The Physics of Laser Plasma Interactions", Westview Press, 2003 